\title{
Um esquema em duas camadas para suporte à mobilidade em redes de sensores sem fio
}

\author{
Filippe C. Jabour Neto, Eugênia Giancoli e Aloysio C. P. Pedroza
}

\begin{abstract}
Resumo-Este artigo propõe um esquema em dois níveis para o suporte à mobilidade em redes de sensores sem fio. Baseado em informações oriundas de algoritmos distribuídos de localização, agentes móveis executam um algoritmo de mobilidade controlada e cumprem a tarefa de sensoriamento na rede apesar da mobilidade aleatória dos nós sensores. Nós apresentamos o modelo de decisão e migração implementado no agente móvel. Foi desenvolvido e validado um simulador computacional e testes foram executados para avaliar a corretude do algoritmo proposto. Os resultados são apresentados e discutidos.
\end{abstract}

Palavras-Chave-Redes de sensores sem fio, mobilidade, agentes móveis

Abstract-This article proposes a two-tier approach for mobility support in wireless sensor networks. Based on informations coming from a distributed localization algorithm, mobile agents run a controlled mobility algorithm and perform the sensor task, despite the random mobility of sensor nodes. We present the decision model implemented in the mobile agent. A computational simulator was developed and validated, and tests were performed in order to evaluate the correctness of the proposed algorithm. The results are showed and discussed.

Keywords-Wireless sensor networks, mobility, mobile agents

\section{INTRODUÇÃo}

Recentes avanços tecnológicos nas áreas de microprocessadores, comunicação sem fio e micro sistemas eletro-mecânicos têm possibilitado a utilização de redes de sensores sem fio (RSSF) [1]. Os sensores são dispositivos de dimensões reduzidas, com restrições computacionais e de comunicação, além de serem alimentados por baterias. Devido às suas dimensões e à impossibilidade de substituição da bateria, o recurso energético é extremamente escasso. Os sensores têm a capacidade de efetuar medições no ambiente próximo a eles. Isto é feito através de uma ou mais funções de sensoriamento existentes no dispositivo. Ao se combinarem em grandes quantidades (centenas ou milhares), os sensores podem formar grandes redes e desempenhar importantes tarefas de aquisição de dados. Uma vez coletados, estes dados são transmitidos aos limites da rede, os nós sorvedouros. Estes enviam os dados para fora da rede para que possam ser processados. Esta tarefa pode se dar nos mais inóspitos e distantes ambientes. Alguns exemplos das inúmeras aplicações das RSSF são o monitoramento de habitats, pesquisas climáticas, acompanhamento médico, monitoramento de estruturas [2]; monitoramento de ambientes de difícil acesso como fundo do oceano, vizinhanças de atividades vulcânicas, territórios inimigos, áreas de desastres e campos de atividade nuclear [3].

As redes formadas pelos sensores funcionam no modo ad hoc. Assim, os nós funcionam como roteadores, encaminhando os pacotes por múltiplos saltos até o destino final [4]. Este destino pode ser um nó sorvedouro ou outro sensor da rede. Os sensores enviam os dados coletados aos nós sorvedouros, que são os pontos de saída da RSSF. É necessário que a rede utilize um protocolo de roteamento. Esses protocolos são definidos como sendo um conjunto de regras pré-estabelecidas, cujo objetivo é construir e gerenciar rotas para a realização do encaminhamento de dados entre os nós da rede [5].

As redes de sensores podem ser classificadas quanto à mobilidade em estáticas ou dinâmicas. Nas estáticas não há qualquer tipo de mobilidade. Nas dinâmicas, podemos ter mobilidade dos sensores, do observador (sorvedouro) ou do fenômeno, ou ainda de dois ou três destes ao mesmo tempo [6]. Uma aplicação freqüentemente encontrada, relacionada à mobilidade, é o rastreamento da movimentação de um agente externo. A rede de sensores, móveis ou estáticos, através de coletas sucessivas, identifica a presença do objeto e estima a sua localização atual e futura.

A mobilidade relativa entre os nós, o sorvedouro e o fenômeno tem conseqüências importantes no funcionamento da RSSF. Diferentemente do que ocorre em redes ad hoc tradicionais, onde os nós móveis são dispositivos utilizados por pessoas, nas RSSF os sensores não estão interessados no fenômeno e se movem independente deste, levados por agentes externos como marés, correntes eólicas ou fluviais ou pela movimentação de seus hospedeiros (animais, veículos, etc). Neste caso, a RSSF deve manter o monitoramento do evento de interesse do observador, independente desta mobilidade [6]. Por outro lado, os protocolos de roteamento para redes $a d$ hoc tradicionais consideram as restrições de banda e energia em patamares compatíveis com dispositivos de computação móvel tais como computadores portáteis (notebooks) ou computadores de mão (palm tops). Como já mencionado, as restrições computacionais e energéticas dos sensores são muito mais rigorosas. Deste modo, os mecanismos tradicionais de descoberta de rotas e recuperação daquelas perdidas devido à mobilidade consomem recursos de uma forma que pode ser excessivamente custosa para os escassos recursos dos sensores.

A motivação para o estudo de mobilidade em redes de sensores está no seguintes cenários: sensores usados na análise de movimento de tornados [6]; sensores conectados a automóveis circulando em metrópoles para estudar as condições do trânsito e planejamento de rotas [6]; sensores flutuando em correntes de rios [7]; projeto da NASA para exploração de Marte, com sensores movidos por ventos [8]; Sensores introduzidos no corpo humano para monitorar condições físicas [9].

Na seção II descrevemos o esquema em duas camadas para suporte à mobilidade. Na seção III é apresentado o modelo 
de decisão dos agentes móveis. O simulador desenvolvido é descrito na seção IV. Nas seções V e VI são apresentados os testes realizados e as conclusões e trabalhos futuros.

\section{ESQUEMA EM DUAS CAMADAS PARA SUPORTE À MOBILIDADE}

Os cenários e ambientes que pretendemos atender nesta etapa do trabalho são aqueles em que os sensores se movem de maneira involuntária pelo campo de interesse. O objetivo central da proposta é manter viáveis o sensoriamento em uma dada região geográfica e a entrega das mensagens aos observadores, apesar da mobilidade de todos os nós da rede. Trata-se de um esquema em dois níveis. O nível inferior é o de interações locais, feito por troca de mensagens, que tratará da localização dos nós e da detecção de chegada e partida de nós sensores. O nível superior é o de troncalização, conduzido por agentes móveis, que procurará manter a eficácia da rede em presença da mobilidade.

\section{A. NIL - Nível de interações locais}

1) Criação de células: Ao ser inicializado, no surgimento da rede como um todo ou na adição posterior de sensores à rede, o nó deve identificar a presença de vizinhos. Observe que, em um ambiente móvel, pode não haver nenhum vizinho em um instante inicial e, posteriormente, o nó pode entrar no raio de comunicação de um ou mais vizinhos. Isto pode se dar através do envio periódico de uma requisição para enviar dados (RTS) do IEEE 802.11 ou através do protocolo IMEP [10]. Ao identificar um ou mais vizinhos, o nó deve aderir a uma das células identificadas.

O protocolo IMEP citado acima destina-se a dar suporte operacional a protocolos de roteamento, protocolos de controle ou outros quaisquer das camadas superiores. Ele incorpora mecanismos de suporte ao monitoramento de enlaces, conectividade de vizinhos, agregação e encapsulamento de pacotes de controle, difusão (broadcast), etc, sempre para ambientes de comunicações sem fio [4]. Nesta proposta, este protocolo será incorporado ao anúncio de chegada ou partida da área de cobertura dos sensores da célula.

O termo célula aqui empregado não se aproxima das células dos sistemas de telefonia móvel ou celular, relacionadas a uma região fixa de cobertura. Está relacionado a um subconjunto de elementos de um sistema, uma unidade vital, cujos elementos se movem de forma independente e cujo sub-conjunto em si, a própria célula, se move em relação às demais. No algoritmo de decisão dos agentes móveis proposto na seção III, basta o nó ter conhecimento dos vizinhos dentro da sua faixa de comunicação, em um dado instante.

2) Localização intracelular: Muitas aplicações de RSSF requerem que os nós tenham ciência de suas respectivas localizações. Em geral, é muito dispendiosa a inclusão de um sistema de localização (GPS) nos sensores [7]. Todos os nós da rede deverão executar um algoritmo de localização. Intanagonwiwat et al. [11] usam exemplos de requisições que podem ser feitas a uma RSSF: "Quantos pedestres são observados na região geográfica X?" ou "Em que direção e sentido o veículo na região Y está se movendo?’. Observamos, então, a importância do conhecimento da localização do nó.

Quando temos mobilidade dos nós, a localização tem que ser atualizada à medida que o nó se desloca. As restrições dos nós da RSSF e da precisão nas medições nos sinais tornam a localização em RSSF mais difícil que a localização de robôs. Entretanto, a alta densidade de nós em uma rede de sensores pode possibilitar uma cooperação no compartilhamento de informações relativas à localização [7].

As RSSF geralmente operam no modo ad hoc. As técnicas de localização adequadas às redes $a d$ hoc se dividem em:

1) Localização Centralizada: Os sensores enviam informações a um ponto central, onde a computação é feita para determinar a localização dos nós. A mobilidade inviabiliza esta técnica em função dos altos custos de comunicação e dos atrasos intrínsecos [7].

2) Localização Distribuída: Cada nó determina a sua localização através de poucas interações com seus vizinhos próximos. Podem ser de dois tipos: a) Baseada no sinal: $\mathrm{Se}$ baseia no instante de chegada (ou variações nele), potência do sinal ou ângulo do sinal recebido. Nas técnicas propostas, os dispositivos necessários tendem a ser sofisticados e caros ou as considerações sobre propagação do sinal tendem a ser pouco realistas. b) Independente do sinal: Se baseia apenas no conteúdo das mensagens. São mais apropriadas às RSSF, devido às restrições de hardware e energia dos nós sensores. As técnicas locais dependem de uma alta densidade de sementes (nós que conhecem sua localização), de modo que cada nó possa escutar várias sementes. Os nós se comunicam e a localização pode ser estimada. Já nas técnicas de contagem de saltos, quando a densidade de sementes é baixa, os nós estimam suas posições com base na localização das sementes e da contagem de saltos até elas (extraída dos anúncios de localização que as sementes propagam pela rede).

Hu e Evans [7] apresentam um esquema onde a posição inicial do nó não é conhecida. Um conjunto de posições é estimado, escolhido aleatoriamente dentre todas as posições possíveis. Dado um conjunto de possíveis posições atuais do sensor no passo $i$, a estimativa do conjunto de posições no passo $i+1$ é formada por escolhas aleatórias dentro dos círculos com centro em cada estimativa anterior e com raio $=\pi \times V_{\max }{ }^{2}$. O nó não tem nenhuma informação sobre seu movimento, apenas a velocidade máxima possível $\left(V_{\max }\right)$. Assim, há um aumento na incerteza da localização do sensor. Esta é a situação mais geral, onde a movimentação pode se dar em qualquer direção e sentido, e a velocidade varia uniformemente entre 0 e $V_{\max }$. Se mais restrições ao movimento forem conhecidas, como sentido do movimento ou velocidade aproximada, estes valores poderão ser refletidos na função de probabilidade das previsões futuras. A partir de localizações reais recebidas de sementes, diretamente ou através de anúncios por parte dos novos vizinhos, o conjunto de previsões é filtrado, de modo que localizações impossíveis sejam excluídas. A execução repetida do algoritmo mantém um conjunto de possíveis localizações do nó.

Barros e Nogueira [12] descrevem e classificam métodos de localização de nós em RSSF. A comparação dos inter- 
valos de chegada de sinais eletromagnéticos com sinais de ultrassom, trocados com nós de referência (que sabem sua localização), possibilita determinar a localização do nó. Os requisitos de hardware são complexos. Outra opção é medir o desvanecimento do sinal ou variações apresentadas por ele próximo à fonte emissora. A comparação de diversos atributos do sinal sendo recebido no momento com padrões conhecidos permite a estimativa de sua posição. Em um ambiente com mobilidade aleatória, será muito difícil gerar estes padrões de referência. O ângulo de chegada do sinal pode ser utilizado na localização. Entretanto, este método requer antenas muito complexas e não se adequa às RSSF. Técnicas que utilizam o infravermelho não podem ser usadas em ambientes externos. Assim, vimos que foram analisados métodos de localização baseados em cronometragem (time-of-arrival e time-difference-of-arrival), em medição de intensidade, em padrão de sinal, direcionalidade, proximidade e conectividade. Estas não se mostraram apropriados aos cenários de nosso interesse por consumirem muitos recursos dos sensores, por demandarem antenas muito sofisticadas ou por se basearem em comunicação por infravermelho.

Diante do exposto, optaremos pela técnica de localização distribuída, independente do sinal, baseada na contagem de saltos (por requerer uma baixa densidade de sementes). Adicionaremos aqui a ação de agentes móveis que atuarão como propagadores das informações de localização das sementes. Aplicaremos a técnica de estimativa de localização e correção (ajustes) [7]. As sementes necessárias na etapa de ajustes das estimativas serão sensores capazes de identificar sua localização (tipicamente sensores dotados de GPS). Agentes móveis terão, dentre outras, a tarefa de disseminar dados de localização gerados nas sementes. Esperamos com isto, combinar sementes de localização, contagem de saltos, informações de localização do substrato de sensores e os próprios agentes móveis para potencializar as informações de localização e refinar as estimativas de localização dos nós da rede.

No algoritmo de decisão dos agentes móveis proposto na seção III, consideraremos que um algoritmo de localização está em execução e que informações de localização do nós estão disponíveis aos agentes móveis na API do nó.

\section{B. NIG - Nível de interações globais}

O NIL (seção II-A) irá manter atualizado o conjunto de vizinhos alcançáveis por um nó e executar o algoritmo de localização dos nós. Diante desta situação, propomos a existência de um nível de interações globais - NIG. Neste nível, será tratado o transporte troncalizado dos dados agregados da origem ao destino. As tarefas deste nível serão desempenhadas por agentes móveis.

Tong et al. [13] argumentam que diante das restrições atuais dos sensores, é muito difícil, senão impossível, confiar apenas aos sensores as tarefas de acesso ao meio, criação e manutenção de rotas, armazenamento e encaminhamento de pacotes, etc. É proposto então que existam alguns dispositivos poderosos que responderão por tarefas críticas da rede. Como em cenários dotados de mobilidade não controlada não se pode garantir que estes super-sensores estarão no lugar certo e na hora certa, argumentamos que tais tarefas especiais devam ser desenvolvidas através da cooperação de agentes móveis, utilizando recursos combinados de sensores homogêneos.

Vahdat e Becker [14] propõem uma técnica que permite a entrega de mensagens apesar da rede não formar o tempo todo um grafo conexo. Adaptando o que prevê a proposta, os agentes móveis armazenam as mensagens até que "encontrem" outros agentes a quem devam encaminhá-las ou até que a rota desejada volte a estar disponível. Shah et al. [15] propõem entidades reais, as Data MULEs, que coletam dados pela rede. A aplicação no nosso caso é direta, diferindo apenas pelo fato da coleta ser feita por agentes móveis.

\section{Modelo DE DECisÃo dos AGENTES Móveis}

Nesta seção descrevemos as bases do modelo de decisão dos agentes móveis, apresentamos o modelo adotado para prever a localização futura do nó sensor e, por fim, mostramos o funcionamento da tomada de decisão dos agentes móveis.

\section{A. Bases}

Os nós sensores se movem pelo campo de sensoriamento; os nós sensores executam um algoritmo de localização que permite a cada um o conhecimento de sua localização geográfica em um dado instante, com um certo erro; os nós estimam a cada instante a posição futura, com base na trajetória seguida até este instante; os nós mantém uma lista de vizinhos alcançáveis em um dado instante; os agentes móveis têm tarefas de sensoriamento a serem executadas e estas contêm as coordenadas da região onde os dados devem ser coletados (região alvo) e as coordenadas da região para onde os dados devem ser trazidos (região de regresso).

\section{B. O modelo de predição de localização dos nós sensores}

Como resultado do algoritmo de localização, o nó computa periodicamente sua posição atual. De posse de duas destas medidas (pontos $P_{i}\left(x_{i}, y_{i}\right)$ e $P_{i-1}\left(x_{i-1}, y_{i-1}\right)$ ), o nó pode computar o coeficiente angular da reta formada por eles $\left(C_{i}\right)$. Este coeficiente angular representa a direção momentânea do deslocamento do nó sensor. O presente modelo considera que o deslocamento seja influenciado em parte pela direção momentânea (equação 1) e em parte por todo o histórico do deslocamento do nó ( $C_{\text {previsto }_{i-1}}$ na equação 2$)$. Deste modo, a previsão de deslocamento estima a direção futura do deslocamento do nó $\left(C_{\text {previsto }_{i}}\right)$ através da média exponencial entre $C_{i}$ e a última estimativa feita $\left(C_{\text {previsto }_{i-1}}\right)$. Na equação 2 , o coeficiente $\alpha$ determina o peso que se deseja dar à última medição $C_{i}$ e, inversamente, à história passada das previsões, $C_{\text {previsto }_{i-1}}$.

$$
C_{i}=\frac{y_{i}-y_{i-1}}{x_{i}-x_{i-1}}
$$

$$
C_{\text {previsto }_{i}}=\alpha C_{i}+(1-\alpha) C_{\text {previsto }_{i-1}}(0 \leq \alpha \leq 1)
$$




\section{Funcionamento dos agentes móveis}

A seguir apresentaremos o modelo de decisão dos agentes móveis. Com base na localização do nó e na estimativa de deslocamento dele, foi desenvolvido o algoritmo de decisão que define os critérios de migração dos agentes móveis. Está migração entre sensores tem como objetivo cumprir a tarefa de coleta de dados (tendo como referência uma região de interesse, chamada região alvo).

Cada nó $N_{i}$ conhece sua posição geográfica com uma precisão $p$ e mantém um conjunto $V_{i}$ de seus vizinhos (seção III-A). Além disso, cada nó estima continuamente sua posição futura (seção III-B). Ao ser inserido no nó origem, o agente móvel $A_{i}$ inicia uma instância do processo de migração em busca do destino, rumo à região alvo. Periodicamente, o agente móvel consulta a posição atual (estimada) pelo seu nó hospedeiro. Se ele já estiver na região alvo, ele coleta os dados de interesse e inicia uma nova instância do processo de migração, rumo à região de regresso. Se o agente móvel não estiver na região alvo, ele identifica entre os vizinhos (incluindo o próprio hospedeiro) qual deles está se deslocando em direção à região alvo, permanecendo no hospedeiro ou migrando para aquele que tiver a previsão de deslocamento mais alinhada ao centro da região alvo (ver algoritmo 1).

Refinando a linha 6 do algoritmo 1 , temos que $A_{i}$ conhece os pontos $A\left(x_{a}, y_{a}\right)$ e $B\left(x_{b}, y_{b}\right)$ que são os vértices opostos da área de interesse (região alvo). Assim, $A_{i}$ determina o ponto $C\left(\frac{x_{a}+x_{b}}{2}, \frac{y_{a}+y_{b}}{2}\right)$ que é o centro da região alvo. Baseado na última medição de posição $P_{i}$ do seu hospedeiro, $A_{i}$

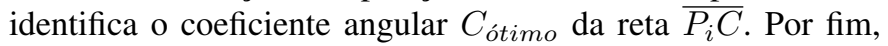
$A_{i}$ determina o coeficiente angular previsto em cada nó que mais se aproxima de $C_{\text {ótimo }}$ e migra para o nó correspondente, se for o caso (ver algoritmo 2).

O presente modelo representa uma simplificação e uma adequação do filtro de Kalman [16] aos escassos recursos de um nó sensor. O esforço computacional é reduzido, de complexidade linear (não dependente do número de nós da rede, mas apenas do número de vizinhos), sendo assim econômico em termos de consumo de energia. O uso de interações locais possibilitam ajustar o tamanho e número de elementos das células do NIL a fim de reduzir o volume de transmissões de dados. O uso de agentes móveis torna dispensável o uso de qualquer algoritmo de roteamento na rede.

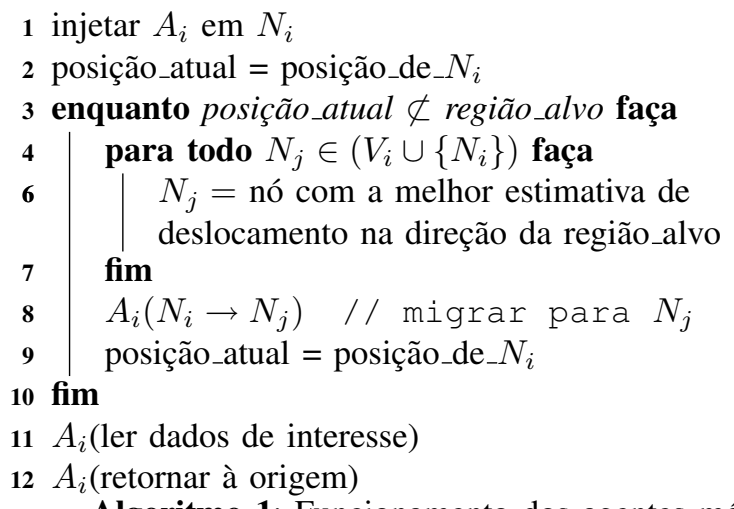

Algoritmo 1: Funcionamento dos agentes móveis

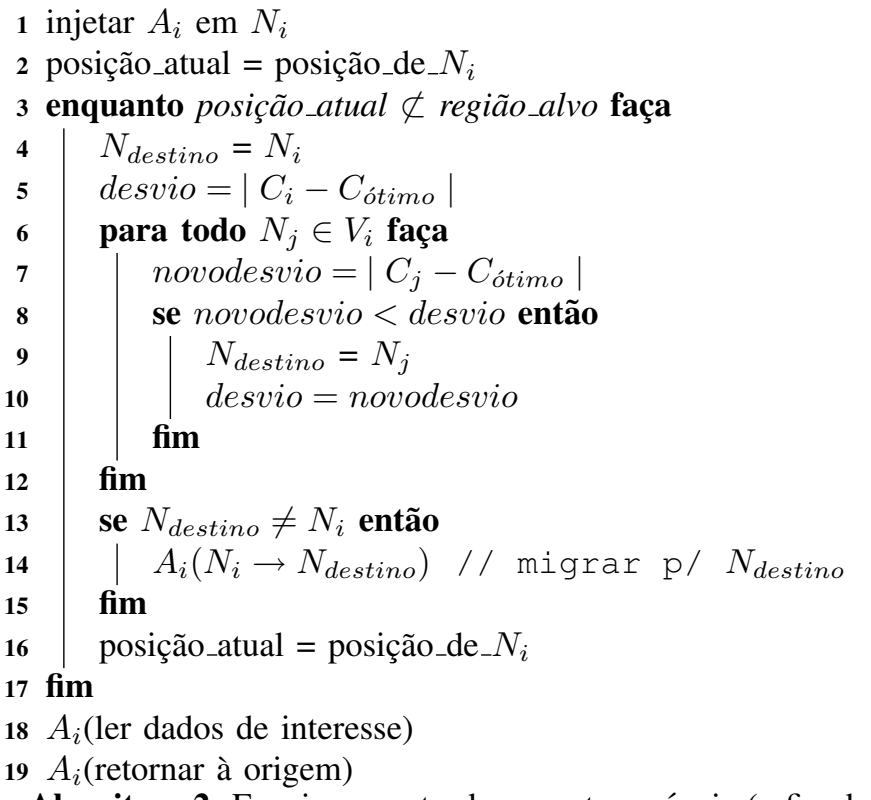

Algoritmo 2: Funcionamento dos agentes móveis (refinado)

\section{Simulador DESEnVolvido}

O simulador $n s$-2 é amplamente utilizado em simulações de redes de computadores, inclusive redes sem fio, redes ad hoc e redes de sensores. O $n s-2$ suporta a mobilidade dos nós e não dá suporte ao uso de agentes móveis. A sua utilização implica na necessidade de implementação de toda a plataforma de agentes. Uma alternativa é o uso TOSSIM [17]. Tratase de um simulador escalável, totalmente compatível com o sistema operacional para sensores TinyOS [18]. Também é amplamente utilizado. Apesar de também não incorporar uma plataforma de agentes, pelo menos uma já se encontra disponível, a plataforma Agilla [2]. Trata-se de um middleware que provê um ambiente de programação de agentes móveis para redes sem fio e que pode ser executado sobre o simulador TOSSIM. Entretanto, não há suporte à mobilidade dos nós no TOSSIM nem na Agilla.

Para avaliar o desempenho do modelo proposto, está em desenvolvimento um simulador específico escrito na linguagem Java. Sua utilização tem a seguinte estrutura:

1) Gerar o cenário de mobilidade na forma de um arquivo texto de entrada. Qualquer trace de entrada com a movimentação dos nós pode ser utilizado, desde que a classe que converte o formato de entrada para o formato do simulador seja customizada. Nas simulações atuais a seguinte sequiência está em uso: (i) Geração de mobilidade através do aplicativo setdest que implementa o modelo Random Waypoint e acompanha a versão padrão do $n s-2$. Aqui são definidos vários parâmetros como área, número de nós, velocidade mínima e máxima, faixas de movimentação e repouso, duração do experimento. (ii) Execução do $n s-2$ para mover os nós segundo o padrão gerado pelo setdest, gerando assim um trace de saída que será o parâmetro de entrada mencionado acima.

2) O simulador lê este arquivo de entrada e incorpora todos os parâmetros existentes para as futuras simulações.

3) Receber mais alguns dados informados pelo usuário: a) Granularidade de tempo (de zero a 12 casas decimais). Tratase de um simulador de eventos discretos onde todos os eventos 
de um passo (segundo a granularidade) são executados antes que o próximo passo esteja liberado para os eventos seguintes. b) Quantos e quais agentes móveis existirão, seus instantes de criação e destruição, as coordenadas da região alvo e da região de regresso (ver seção III) e o nó hospedeiro inicial. c) Raio de cobertura da comunicação de cada nó.

4) Executar a simulação, movendo os nós conforme descrito; executar a predição de localização dos nós móveis conforme seção III-B e executando o algoritmo da seção III-C.

$\mathrm{Na}$ etapa atual, a comunicação entre os nós, envolvendo a consulta aos vizinhos e a migração dos agentes é considerada ideal, sem perdas e colisões.

O resultado do algoritmo de localização é considerado disponível, de modo que cada nó gera periodicamente uma estimativa de localização extraída da localização real \pm um erro especificado.

\section{A. Validação}

Os traces de entrada foram analisados e comparados com arquivos intermediários de depuração gerados pelo simulador. Estes arquivos intermediários que parametrizam as simulações reproduzem exatamente os traces de entrada. Foi criado um relógio central da simulação e através de técnicas de exclusão mútua certificou-se que os vários fluxos de execução (Threads) que implementam os nós e os agentes móveis operam em sincronismo.

\section{TESTES REALIZADOS}

\section{A. Trajetória estritamente crescente}

A primeira etapa de testes buscou validar o algoritmo de predição de localização dos nós (seção III-B). $\mathrm{O}$ valor de $\alpha$ na equação 2 foi fixado em 0,5 , ou seja, a última medição e última estimativa (histórico) têm o mesmo peso. Foi gerada uma trajetória dita real, de um nó, baseada no seguinte padrão: o nó parte da origem e as coordenadas $x$ e $y$ são estritamente crescentes segundo as equações 3 .

$$
\begin{aligned}
& x_{i+1}=x_{i}+\delta, y_{i+1}=y_{i}+\delta^{\prime} \\
& \delta, \delta^{\prime} \in \mathbb{R}, 0<\delta, \delta^{\prime} \leq 10
\end{aligned}
$$

Além da estimativa do coeficiente angular (direção) do deslocamento, foi estimada, da mesma forma, a velocidade de deslocamento. Ou seja, se estimou a direção e o módulo do vetor velocidade em cada passo da simulação.

Nesta situação, como a trajetória apresenta reduzida variação de direção e velocidade escalar, o previsor acompanha de forma satisfatória a trajetória real (figura 1 e tabela I). Estas previsões, como argumento de decisão para os agentes móveis, irão produzir migrações corretas na busca da região alvo.

\section{B. Trajetória do tipo Manhattan}

Neste caso, buscou-se avaliar o algoritmo de predição de localização dos nós em um segundo cenário, com mobilidade menos regular do que a usada na seção V-A. O parâmetro $\alpha$ foi mantido em 0,5 . A trajetória real segue o modelo

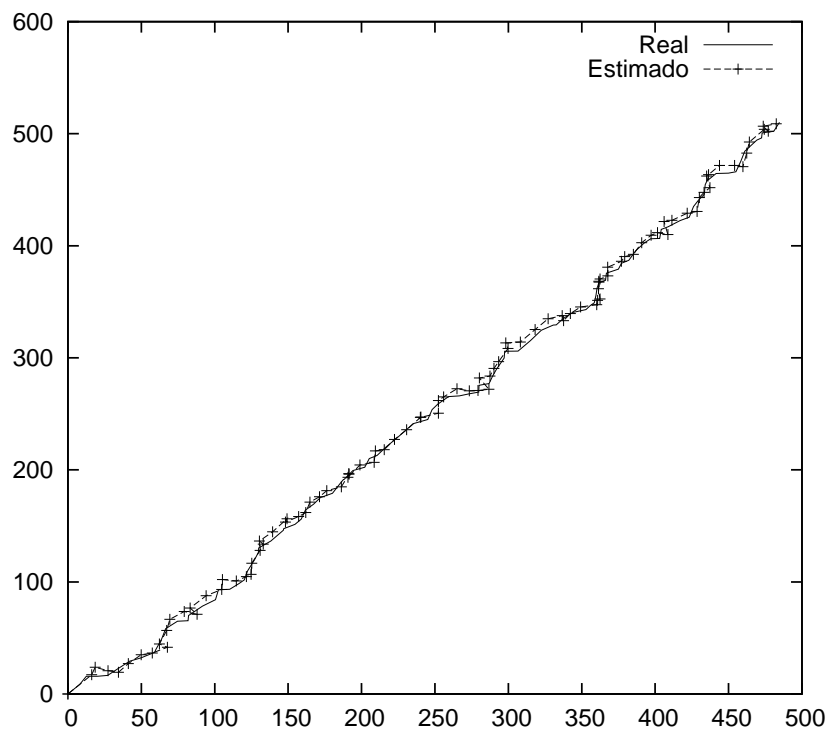

Fig. 1. Predição de localização do nó - trajetória com menor variação

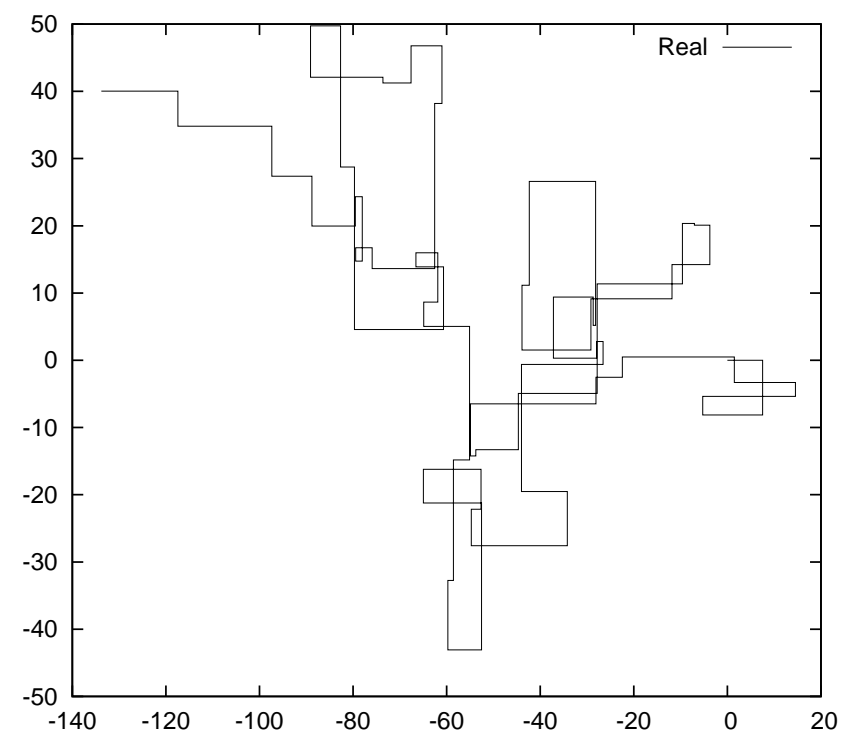

Fig. 2. Trajetória real do tipo Manhattan

Manhattan [19]. Mais uma vez usou-se o método para estimar direção e módulo da velocidade. Esta trajetória apresenta uma maior variação de direção, com mudanças de $90^{\circ}$. A cada movimento, a coordenada sorteada é modificada em uma faixa de -30 a $30 m$ (3 vezes mais que no caso anterior). Observase na figura 2 que a trajetória se desloca por toda a área do gráfico, apresentando mudanças completas de direção. Mais uma vez o previsor obtém sucesso nos resultados gerados (figura 3 e tabela I) e estes permitirão a migração acertada dos agentes móveis. 


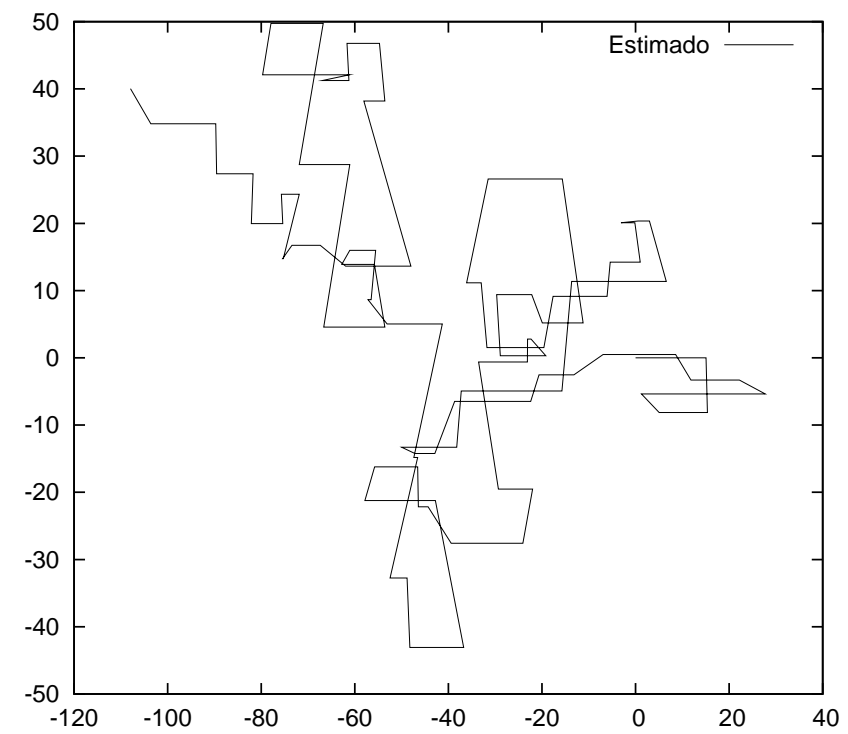

Fig. 3. Trajetória prevista para o modelo Manhattan

TABELA I

ESTATÍSTICA DAS PREVISÕES (METROS)

\begin{tabular}{||l||c|c||}
\hline \multicolumn{1}{||c||}{} & Trajetória regular & Trajetória Manhattan \\
\hline \hline Erro mínimo & 0,74 & 0,62 \\
\hline Erro máximo & 11,13 & 32,5 \\
\hline Erro médio & 4,67 & 13,59 \\
\hline Desvio padrão & 2,35 & 7,7 \\
\hline
\end{tabular}

Considerar que na trajetória Manhattan os deslocamentos são em média 3 vezes maiores que na trajetória regular (equivale ao triplo da velocidade)

\section{CONCLUSÕES E TRABALHOS FUtUROS}

A proposta de suporte à mobilidade baseada em dois níveis permite que as tarefas sejam divididas e se tornem menos dispendiosas aos nós da rede. As interações locais envolverão um pequenos número de nós e o uso de agentes móveis no nível global permitirá a execução da tarefa de sensoriamento apesar da mobilidade aleatória dos nós sensores.

O modelo de predição de localização apresentado é eficiente e suficiente para servir de base de decisão aos agentes móveis na busca da região alvo a ser sensoriada. As simulações mostram a eficácia do método de localização com erro médio variando de 4 a $13 m$ (reduzido se comparado à faixa de cobertura de rádio dos sensores e das áreas e velocidades simuladas). O referido método é de complexidade computacional constante, o que acarreta baixo consumo de ciclos de CPU e, conseqüentemente, de energia. Isto é fundamental em RSSF.

O algoritmo de decisão dos agentes móveis é de complexidade linear, dependente apenas do reduzido número de vizinhos do nó hospedeiro. De posse de boas previsões de deslocamento dos nós, os agentes móveis poderão se manter sempre nos nós com maior probabilidade de deslocamento na direção da região alvo e, posteriormente, na direção da região de regresso.

O simulador desenvolvido implementa o modelo de previsão e o algoritmo de decisão dos agentes móveis, possibilitando a análise das proposta em um ambiente ideal de comunicação.
Como trabalhos futuros serão executadas análises do módulo de previsão de localização com outros modelos de mobilidade; análises do módulo de previsão de migração em presença de erros de localização (presentes em todos os métodos de localização, sobretudo os indiretos, que são adequados às RSSF); avaliação do algoritmo de decisão dos agentes móveis em diversos cenários de mobilidade; introdução de um modelo de comunicação mais realista no simulador, com base em modelos estocásticos de perdas nos níveis físico e de enlace de dados; análise da mobilidade em 3 dimensões.

\section{REFERÊNCIAS}

[1] Ruiz, L. B., Correia, L. H. A., Vieira, L. F. M., Macedo, D. F., Nakamura, E. F., Figueiredo, C. M. S., Vieira, M. A. M., Bechelane, E. H., Camara, D., Loureiro, A. A., Nogueira, J. M. S., DA Silva JR., D. C., E Fernandes, A. O. Arquiteturas para redes de sensores sem fio. XXII SBRC (Maio de 2004). Brasil.

[2] FoK, C.-L., Roman, G.-C., E Lu, C. Rapid development and flexible deployment of adaptive wireless sensor network applications. 25th IEEE International Conference on Distributed Computing Systems (ICDCS'05) (2005), 653-662.

[3] Pereira, M. R., De Amorim, C. L., e de Castro, M. C. S. Tutorial sobre redes de sensores, 2001. Disponível em: http://magnum.ime.uerj.br/cadernos/cadinf/vol14/3-clicia.pdf.

[4] Corson, S., E MACKer, J. Mobile ad hoc networking (MANET): Routing protocol performance issues and evaluation considerations. Request for Comments: 2501 (January de 1999).

[5] Campos, C. A. V., e de Moraes, L. F. M. Caracterizando a mobilidade de usuários e avaliando seu impacto no roteamento das redes móveis ad hoc. Em WCSF (2004), p. 127.

[6] Tilak, S., Abu-Ghazaleh, N. B., e Heinzelman, W. A taxonomy of wireless micro-sensor network models. ACM SIGMOBILE Mobile Computing and Communications Review 6, 2 (2002).

[7] Hu, L., E Evans, D. Localization for mobile sensor networks. MobiCom '04 (2004), 45-57. ACM.

[8] Antol, J., Calhoun, P., Flick, J., Hajos, G. A., Kolacinski, R., Minton, D., OWens, R., E PARKer, J. Low cost mars surface exploration: The mars tumbleweed. NASA Langley Research Center. NASA/TM-2003-212411.

[9] Loureiro, A. A. F., Nogueira, J. M. S., Ruiz, L. B., De Freitas Mini, R. A., Nakamura, E. F., e Figueiredo, C. M. S. Redes de sensores sem fio. XXI SBRC (2003), 179 - 226. Brasil.

[10] Corson, M. S., Papademetriou, S., Papadopoulos, P., Park, V., E QAYYUM, A. An internet MANET encapsulation protocol (IMEP) specification. INTERNET-DRAFT (7 de Fevereiro de 1999). IETF MANET Working Group.

[11] Intanagonwiwat, C., Govindan, R., E Estrin, D. Directed diffusion: a scalable and robust communication paradigm for sensor networks. Em MobiCom '00: Proceedings of the 6th annual international conference on Mobile computing and networking (New York, NY, USA, 2000), ACM Press, pp. 56-67.

[12] Barros, R. Q., E NogueIRA, J. M. Estudo de métodos de localização de nós em redes de sensores sem fio. VII semana da pós-graduação DCC - UFMG (2003).

[13] Tong, L., ZhaO, Q., E AdiReddy, S. Sensor networks with mobile agents. Em IEEE Military Comm. Conf. (Oct de 2003). Boston, MA., http://acsp.ece.cornell.edu/.

[14] VAHDAT, A., E BECKER, D. Epidemic routing for partially-connected ad hoc networks. Technical Report CS-200006, Duke University, April 2000.

[15] Shah, R. C., Roy, S., Jain, S., e Brunette, W. Data mules: Modeling a three-tier architecture for sparce sensor networks. IEEE SNPA Workshop, May 2003.

[16] Kalman, R. E. A new approach to linear filtering and prediction problems. Transactions of the ASME-Journal of Basic Engineering 82, Series D (1960), 35-45.

[17] LEVIS, P. TOSSIM: Accurate and scalable simulation of entire TinyOS applications. Proceedings of the First ACM Conference on Embedded Networked Sensor Systems (SenSys 2003), November 2003.

[18] Tinyos. http://www.tinyos.net/. Acesso em junho de 2007.

[19] Selection procedures for the choice of radio transmission technologies of the umts (ts30.03 v3.2.0). TS 30.03 3GPP, Abril de 1998. 